\title{
Original
}

\section{Pulse Train Analyzer for Laboratory Use and Its Application to The Anticipation of Magnet Relay Breakdown}

\author{
by \\ Ryuji IGARASHI $^{\dagger}$, Yukio IwaYA ${ }^{\dagger}$ and Masato $\mathrm{SAKATA}^{\dagger}$
}

\begin{abstract}
A pulse train analysis is reported here from the viewpoint of both a hardware and a software. In hardware part, an interface board is specifically designed which can administer the height and the timing of the pulses of a pulse train. The front-end of the hardware system is a Wilkinson-type ADC. The crude data taken in by the hardware are pulse height and arrival time of respective pulses. The software part reconstructs the data into pulse height distribution, time interval distribution, count rate representation, direct representation of pulses along the time elapsed, frequency distribution $f(n)$ of pulse count $n$, and such statistical moments as $\mu, \sigma, S, K$ of $f(n)$. Statistical tests can be practiced as well. The system is actually applied to the anticipation of magnet relay breakdown.
\end{abstract}

Key Word: Pulse Train, Pulse Processing, Micro Computer, Interface, ADC, Prediction, Breakdown Anticipation, Insulator, Magnet Relay, Statistical Treatment

\section{Introduction}

Some of the physical phenomena are measured by pulse-type detectors ${ }^{1)}$. In such measurements a pulse train having pieces of information in pulse height and in occurrence time is formed. There are a lot of research work concerning the processing of pulse trains in the field of nuclear radiation detection $^{2-6)}$, physiology ${ }^{3,7)}$, photon counting ${ }^{8)}$, electrical insulation ${ }^{9-12)}$, and acoustic emission ${ }^{13)}$. In laboratories computers are used to take pulse data ${ }^{2,4-7,10,11,14,15)}$ and the computer can rebuild them into Pulse Height Distribution (PHD) ${ }^{4-6)}$, Time Interval Distribution (TID) ${ }^{3,8,10,11,14,16)}$, or into Count Rate $(\mathrm{CR})^{2)}$. We have developed a micro computer based system specifically designed for taking pulse trains. The feature of our system is that it can successively take in the incoming pulses without skipping any one of them. A limitation is put to our system only when the interval of the adjacent pulses is shorter than the total processing time of the system. It can process the crude information into PHD, TID, CR, statistical quantities and visual representation ${ }^{16)}$. In the statistics part of the system, a frequency distribution $f(n)$ of pulse number $n$ in a unit time is constructed from the crude data. The system can derive moments of $f(n)$ and can display them. Also

Received July 31, 1995

† Department of Electric and Electronic Engineering, Akita University, 1-1 Tegata Gakuencho, Akita City, Akita, 010, Japan 
it can implement statistical tests between two adjacent $f(n)$ s along the elapsed time of the pulse

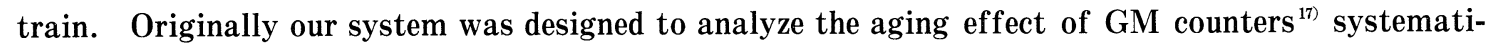
cally. The hardware part of the system is based on a commercially-available, Wilkinson-type Analogto-Digital Converter (ADC) and an Interface (IF) board designed exclusively for controlling ADC.

A timer module consisting of 6-byte counter is one of the features of our sysytem. With this counter we can trace a pulse train up about to $10^{10}$ counts.

An application example of the system is described concerning the anticipation of the breakdown of a magnet relay. Breakdown phenomena may be regarded as aging processes. Therefore, our system can be applied to the anticipation of a magnet coil breakdown. The immediate phenomenon observed in the course of coil deterioration is the occurrence of Partial Discharge (PD) pulse which depends on the time elapsed. A PD is a small discharge allowed to appear before the complete breakdown of insulation materials. There were a lot of reports about the PD ${ }^{18-21)}$. The occurrence of PD pulses is a stochastic phenomenon and, to some extent, the occurrences of the PD pulses are independent with one another. The independence of repective PDs are evaluated by number distribution ${ }^{22)}$ or by TID representation ${ }^{12,23)}$. Several cases were reported in which the occurrences of pulses are not independent to each other from the viewpoint of TID $^{24-28)}$.

2. Hardware System

\section{1 Overview of the system hard-} ware

A conceptual description of the system hardware is illustrated in Fig. 1. The front end of the system consists of a Linear Amplifer (LA). Analog pulses sent our of a detector are fed into a Wilkinson-type ADC through the LA. After the conversion of the pulse height, the data are sent to an IF board which is a gateway to a Pulse Height Analysis (PHA) system. Arrival time of a pulse is measured by counting Clock (CLK) pulses in a Time Counter (TC) board which has a CLK generator. Binary data from both PHA and TC board are combined into a packet and then input to a bank RAM. After the bank RAM is filled with the data, the computer stores them into an extemally attached Hard Disk (HD) memory.The IF and the CPU repeat the above

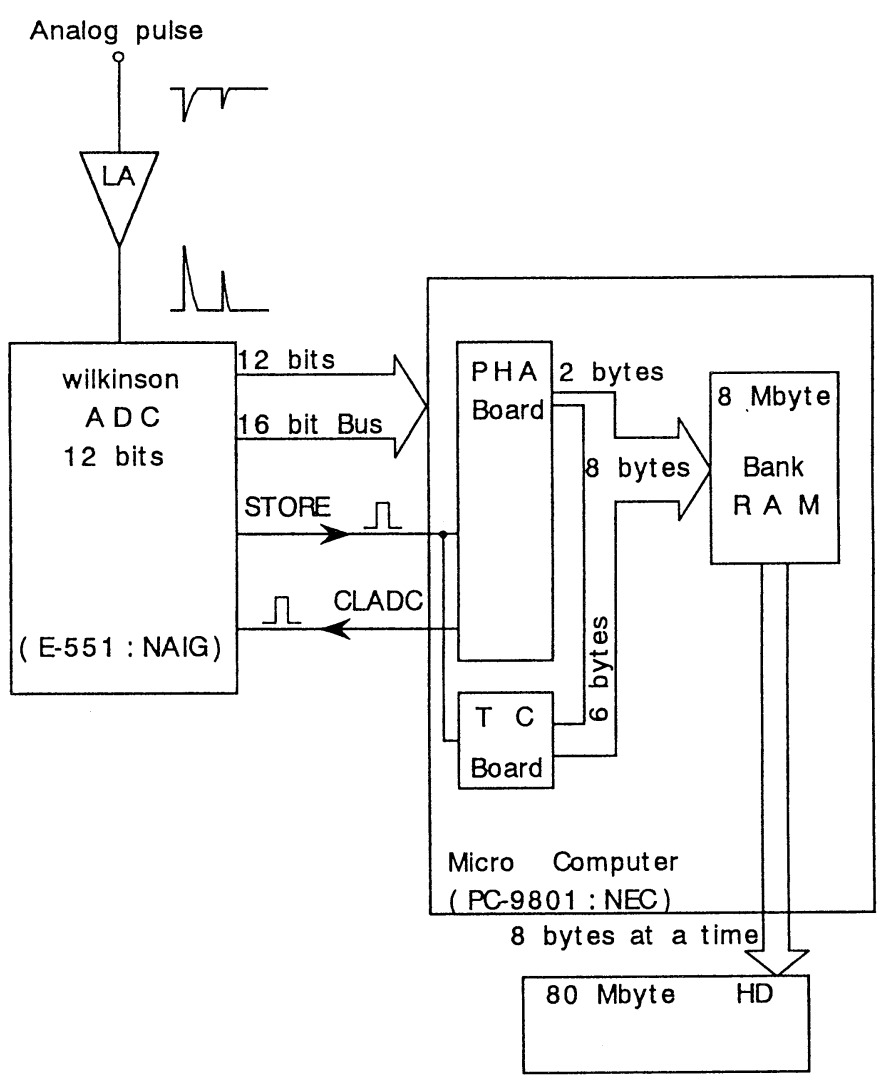

Fig. 1 The illustration of the pulse data handshake between the micro computer and the ADC. 
operation until total input number $N_{\mathrm{P}}$ amounts to an initially set count.

2. 2 Description of each part

2. 2. 1 Handshake between $A D C$ and computer

An analog pulse fed out of the LA is input to the ADC (E551, NAIG) and the pulse height is transformed to a binary data. The data length is 12 bits. The 12-bit-long data is processed at a time as 2-byte data on the IF board. Therefore, the micro computer employed (PC9801VM 21 , NEC) can reduce the handshake time between the CPU and the ADC. After the conversion, the ADC sends an acknowledge-

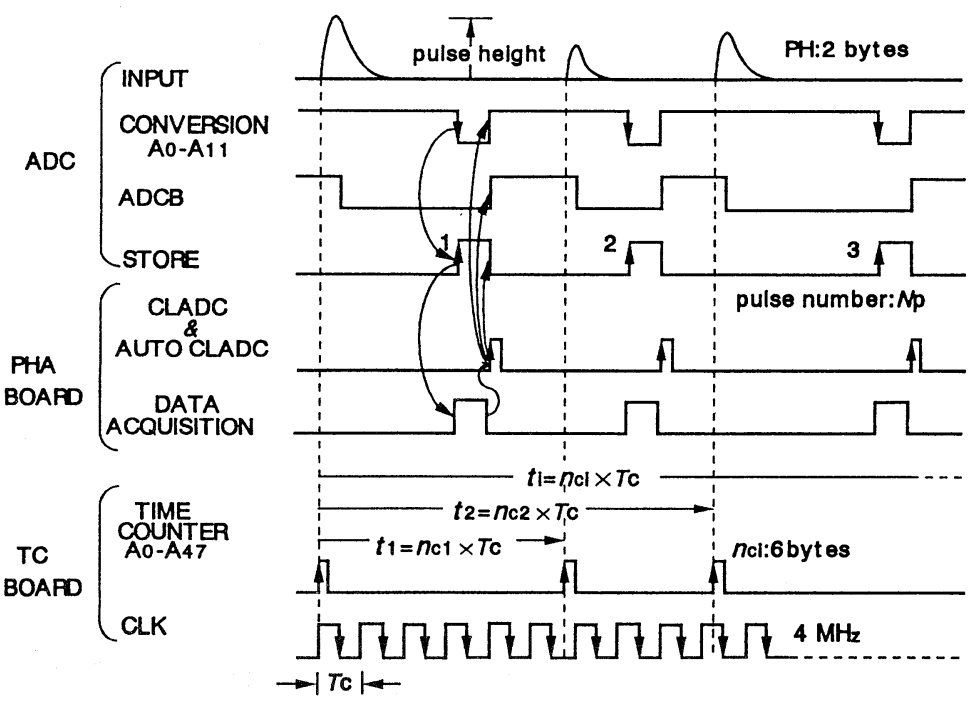

Fig. 2 The illustrative explanation of the relationship between repective signals which administer the handshake of the interface system. ment signal (STORE signal) to the PHA board, and the boared receives the 12-bit data succeeding to the STORE signal identification. The signal stream is described in Fig. 1 and the relationship among signals is illustrated as a time chart in Fig. 2. The ADC becomes inoperative until it is reset to the initial state. The reset is executed by a Clear ADC (CLADC) pulse. This pulse is sent out of the PHA board after the computer stored the 2-byte data into the bank RAM. The RAM is installed in order that system can store as many data as possible in as short a time as possible without skipping any data. An 8-Mbyte RAM board is installed in the computer and, with such allocation as shown in Fig. 3, 63

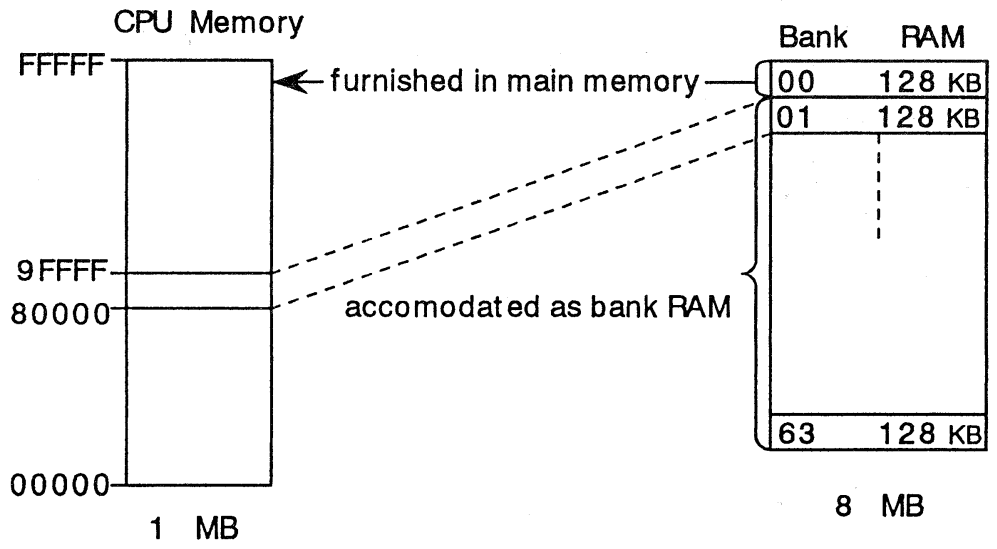

Fig. 3 Installment of a bank RAM in order to expand the CPU memory. 
banks become available. On receiving the CLADC, the ADC again becomes ready for the next pulse input, thus continuing hand-shakes up to initially set count. During through the hanshake operation, the computer can check and count the number $N_{\mathrm{P}}$ by receiving STORE signals. This enables the system to observe the cumulative number of input analog pulses.

\section{2. 2 Arrival time measurement of input pulses}

We can measure with our system both a pulse height and an arrival time of the pulse. Arrival time here means the time elapsed from initial trigger pulse. The quantity actually measured is the number $n_{\mathrm{c}}$ of the CLK pulse. Therefore, for $i$-th input pulse, the arrival time $t_{\mathrm{i}}$ is determined by $t_{\mathrm{i}}=n_{\mathrm{c} \text { i }} \times T_{\mathrm{c}}$, where $T_{\mathrm{c}}$ is the period of the CLK pulse. A total scheme is illustrated in Fig. 2. A counter LSI prepared for the system is $3 \times 16$-bit-long, programmable one ( $\mu$ PD71054, NEC) which works up to $8 \mathrm{MHz}$. In our setup the CLK frequency is fixed at $4 \mathrm{MHz}$, hence $T_{\mathrm{c}}$ being $250 \mathrm{~ns}$. Two counter LSIs are installed parallel onto the TC board so that the 16-bit CPU can handle the counter data at a time and can reduce the handshake time. The reason the parallel access is practiced is that the data bus of the counter LSI is 8 bits and, to match it to the 16-bit bus, the counter LSIs must have been furnished parallel. The connection scheme of the programmable counter is illustated in Fig. 4. In order to enable the computer a 16-bit access, two counter LSIs are programmed to work with 8-bit count action under the mode-2 operation of the counter LSI. As represented in Fig. 4, one counter is connected to the lower data bus, and the other to the upper data bus.

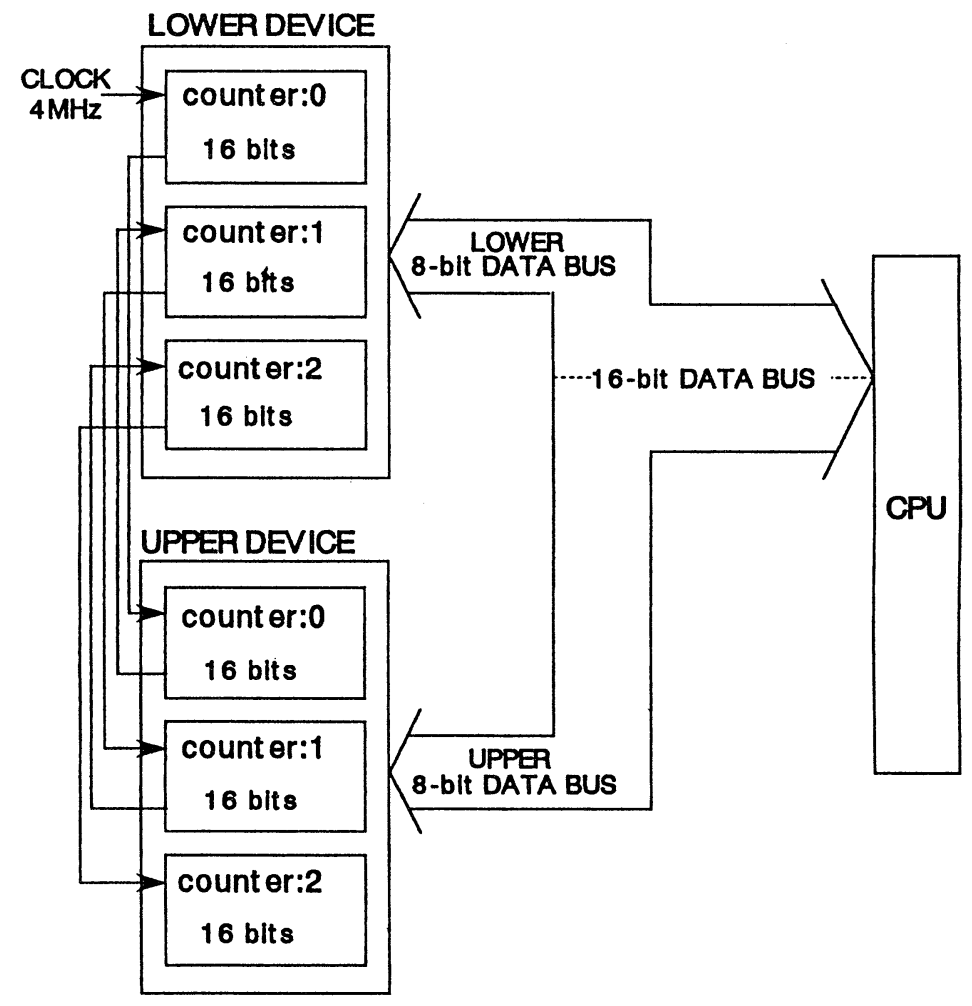

Fig. 4 Simplified explanation of the programmable counter arrangement which enables the long trace of the pulse train. 
Three separate counters in the counter LSIs are altemately connected sequentially as illustrared in Fig. 4. This sequential connection enables the counter to implement a 16 bits $\times 3=48$-bit-long CLK count action.

Immediately after the input of the initial analog pulse, the counter begins to work and continues its operation until it receives the final pulse. Counter data, when read by CPU, are latched, and thereafter, the latch is released. At the instant the latch action starts, the TC board receives the index pulse corresponding to the input analog pulse. This means that the computer can construct Time Interval (TI) data $t_{\mathrm{i}}-t_{\mathrm{i}-1}$ as explained in Fig. 2. Since the counter LSI is $3 \times 16=48$-bit long, data length of $n_{\text {c i }}$ explained in Fig. 2 becomes 6 bytes. Actually, the data length stored into the bank RAM is $2+6=8$ bytes because 2 -byte PHA data and 6 -byte counter data are combined with each other. With the present preparation the bank RAM can store $\{128 \mathrm{k}$ bytes $\times 63$ banks $\} / 8$ bytes $=\{(128 \times 1024) \times 63\} / 8=1.032192 \times 10^{6}$ packet data which corresponds to $1.032192 \times 10^{6}$ analog pulses. The storage capacity depends on the RAM capacity. Since we installed an 8-Mbyte RAM into the computer, the total data we can treat become $\{128 \mathrm{k}$ bytes $\times 63$ banks $\}$ kbytes.

One of the characteristics of our system is the successive introduction of incoming analog pulses without skipping any one of them. However, the feature depends on the system processing time because the time acts as a dead time of the system. The dead time gives a limitation to the system performance. In case an interval between successive pulses is shorter than the dead time, a skipping of a pulse takes place. The dead time, as seen from Fig. 2, is the time from an analog pulse input to the output of a clear ADC (CLADC) pulse which is sent out of the PHA board. This cycle consists of the conversion time of the ADC, a CPU processing time needed to fetch the CLK count data, and the time to send the CLADC pulse to the ADC after storing the data into the bank RAM. The conversion time of the ADC depends on the pulse height of the input signal. With the present model the full height of the input signal is converted to 1024 channels. Conversion time per channel is 0.02 $\mu \mathrm{s}$. Therefore, with the full height, the ADC dead time becomes $1024 \times 0.02=20.5 \mu \mathrm{s}$. The computer we employed needs $15 \mu$ s to execute the above cycle. Accordingly the system dead time becomes $35.5 \mu \mathrm{s}$ at the maximum. This value is short enough to apply our system to the detection of PD pulses from magnet relays.

\section{2. 3 Utilization of externally installed hard disk memory}

One of the goals of our development is to achieve as longer storage of analong pulse data as possible. We installed a Hard Disk (HD) unit to realize this. The memory capacity of the HD emplpyed (MH-8, CRC) is 80 Mbytes. Basic idea consists of a hybrid storage of the data using a bank RAM and a HD. The bank RAM makes fast storage possible and the HD helps the system store a large amount of data. However, as seen from Fig. 5, the CPU becomes inoperative to the handshakes between the ADC and the IF during the transfer action from the RAM to the HD. The situation is described conceptually in Fig. 5. As is seen from Fig. 5, the CPU also becomes inoperative during the transfer action from the RAM to the HD. Even in the period in which the CPU can not respond to the ADC, an installment of a specifically designed IF board makes it possible to practice virtual handshakes between the ADC and the IF board by sending CLADC pulses automatically from the IF board (Auto CLADC). By this procedure we can contiune to trace the analog pulse. The count number of the analog pulse is denoted $N_{\mathrm{p}}$ as described in $\S$ 2.2.1. The ADC operation is controlled by the hardware installed in the IF board. Since the ADC is used as a gateway to 


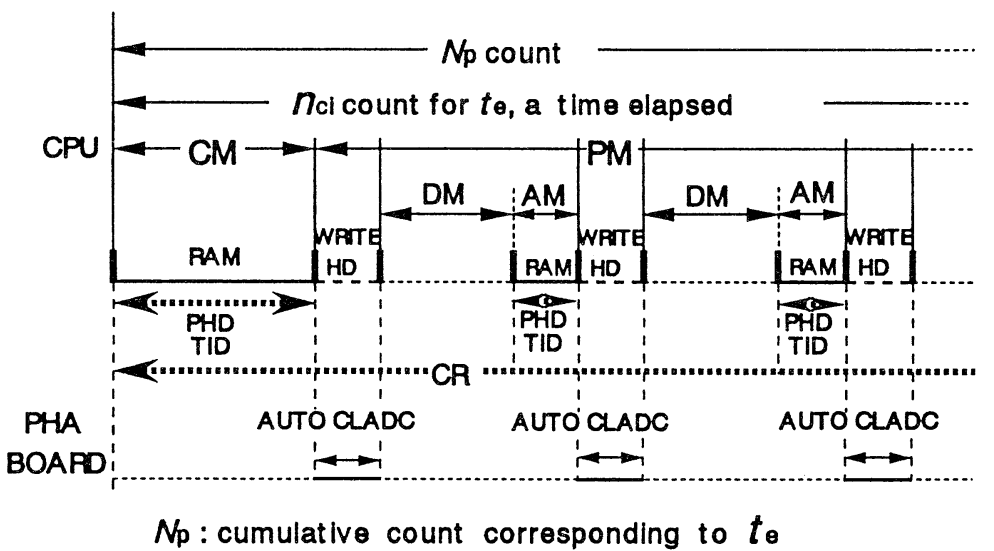

Fig. 5 Description of data transfer from a RAM to an externally attached Hard Disk (HD) memory. In order to remove inoperative period of the ADC during the data transfer, the PHA board supervises independently the handshake without the assistance of the CPU (Auto CLDC).

pulse height analysis, the board is named a PHA board.

Owing to the PHA board we specifically designed, the system can trace the input analog pulses even when the CPU does not store them. By using this function we can store part of the data of the pulses while the system traces the count number of the pulse train. This saves greatly the memory capacity so that the system can extend the upper limit of the traceable number of the pulses. Here, an attention should be paid that there are pieces of duration in which the system does not store the ADC data after the continuous introduction of them. We call such duration as Partial Measurement (PM). The PM further consists of Dummy Measurement (DM) and Active Measurement (AM). During the DM the processor does not store ADC data. However, the pulse trace is going on and $N_{\mathrm{p}}$ count proceeds. After passing the DM period, the processor begins to store ADC data to the bank RAM. This is the AM period. When the AM action is over, the processor stores the RAM data into the HD and resets the RAM area so that the RAM can receive data without an overflow for the next AM period. At the initial operation of the analog pulse input preceding to the PM, the data are stored into the RAM up to allowable limit of the memory. The full length is, as was mentioned in $\S 2.2 .2,1.032192 \times 10^{6}$ pulses. During the above operation, all pulses are introduced continuously in case the pulse interval do not exceed the system dead time. Therefore, we call this operation a Continuous Measurement (CM). The CM procedure becomes, for example, extremely useful when an aging phenomenon shows a rapid change in the initial duration.

\section{Data Analysis}

In terms of the analysis of the data, the software burdens most part of our system. The crude data provided directly from the hardware are the pulse height and the arrival time $t_{\mathrm{i}}$. All other quantities we shall describe in the following are derived from the crude data. 
The method in order to derive CR, PHD, TI, TID and $f(n)$ is conceptually represented in Fig. 6, where $f(n)$ expresses a frequency distribution of analog pulse count $n$ per arbitrary time $t$. As was mentioned in $\S 2.2 .1$, the system can count the number $N_{\mathrm{p}}$ of input analog pulses. If we check the number every $t_{\mathrm{p}}$ seconds, then we can obtain the CR (= counts $/ t_{\mathrm{p}}$ ) and can illustrate the CR variation. As we described in $\S 2.2 .2$, the time interval $T I_{\mathrm{i}}$ in the $i$-th sequence is immediately obtained by $T I_{\mathrm{i}}=t_{\mathrm{i}}-t_{\mathrm{i}-1}$. Therefore, we can construct TIDs and see the temporal variation of them by processing TI evey $n_{\text {p }}$ pulses. Here, one point which an attention should be paid to in Fig. 6 is the different length of each $n_{\mathrm{p}} . \quad N_{\mathrm{p}}$ and $n_{\mathrm{p}}$ represented in Fig. 6 not only express pulse counts but they also are measures to estimate $t_{\mathrm{e}}$, the time elapsed in the experiment. In a pulse producing sample, an aging of the sample depends on the preceding history of the pulse occurrence. In that instance the number of pulse count is a better measure of the aging than $t_{\mathrm{e}}$ itself because the sample property is considered unchanged without pulse occurrence. However in a random pulse train, the same durations does not accompany the same $n_{\mathrm{P}} \mathrm{S}$ because different time intervals constitute the $n_{\mathrm{P}}$. This is why different lengths are assigned to respective $n_{\mathrm{p}} \mathrm{S}$ in Fig. 6.

In our system the software can prepare frequency distributions $f(n)$ for further statistical analysis. As mentioned above, $n$ is a count per arbitrarily set $t$. By rearranging the frequency of $n$ during $t_{\mathrm{g}}$ we can construct an $f(n)$. Repeating this procedure over several $t_{g} \mathrm{~s}$, we can arrange time dependent $f(n)$ s and can study the aging by observing $f(n)$. Concretely, we can derive such

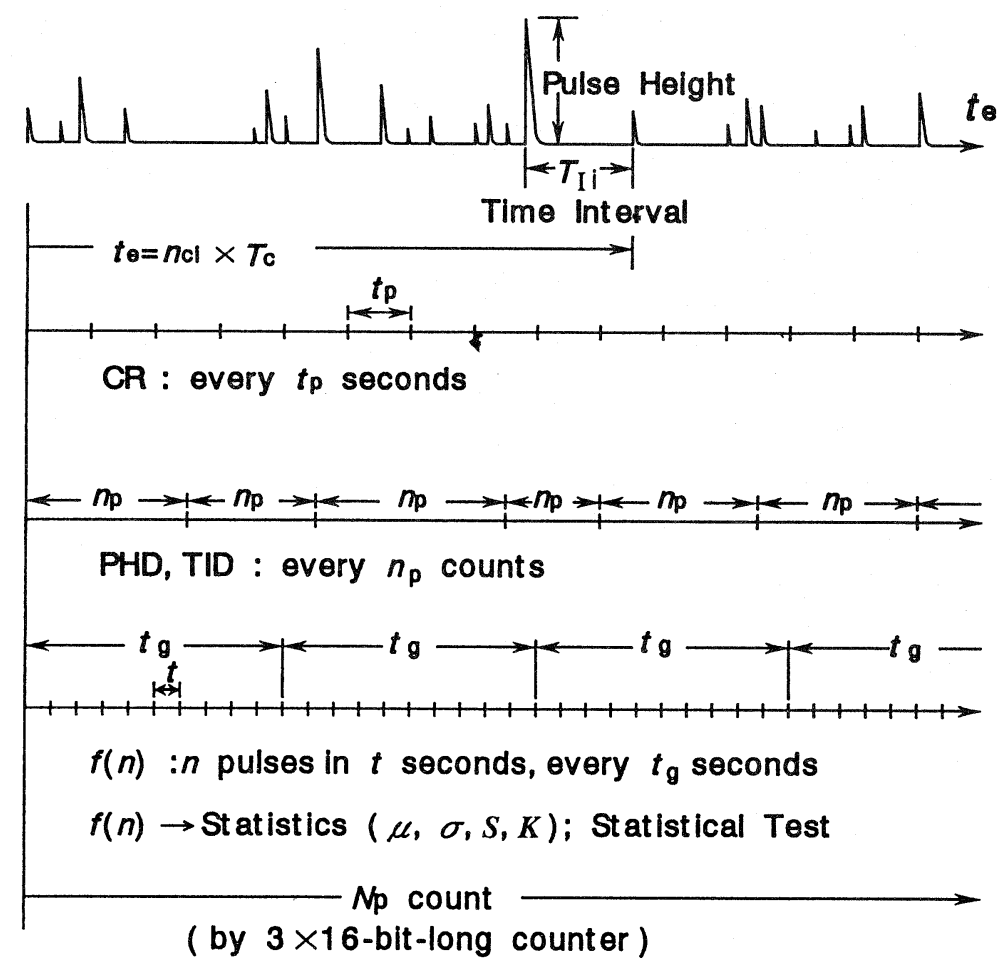

Fig. 6 Illustrative explanation of the data processing and analyses of the pulse train. 
statistical quantities as mean $(\mu)$, variance $\left(\sigma^{2}\right)$, skewness $(S)$ and $\operatorname{kurtosis}(K)$ from $f(n)$ and can represent their temporal variations. Statistical tests can also be implemented between two different $f(n)$ s. The result of the test becomes also a measure to evaluate whether or not the aging comes to appear. The statistical test can be practiced for mean discrepancy and variance.

We may construct an overview about a pulse train by data analyses stated above. In case a rapid variation appears in the pulse train in a short duration, however, the representation of the pulse train by individual pulse becomes preferable. Therefore, we propose a illustrative description of each pulse by its height, its time elapsed and the cumulative count $N_{\text {p }}$ of the pulses, where $N_{\text {p }}$ corresponds to the elapsed time $t_{\mathrm{e}}$. Since there are three kinds of quantities, we employ a ThreeDimensional (3D) representation. Also from the 3D data we can rearrange a Two-Dimensional (2D) representation which superimpose two combinations of two quantities. An example and a detailed explanation are given in the following section.

\section{Application to Breakdown Anticipation}

In this section an application example of the pulse analysis system to the anticipation of insulation breakdown is described. A magnet relay is chosen as an example. Once it comes to breakdown, it gives a crucial influence, even damage, to electrical notworks or to a power station. Therefore, an anticipation is necessary to prevent electrical systems from a failure.

An insulator gives partial discharges (PDs) even well before its breakdown ${ }^{10,11,15,29-32)}$. With an appropriate procedure, the PD pulses can be taken in to our system. In the following subsections the separation of PDs from a magnet coil is described first. Then the formation of pulse train, the input of the pulses into the computer, and the results of the analyses of the crude data are described.

\section{1 Detection principle}

An outline of how to pick up the PD pulses from a amagnet coil is conceptually shown in Fig. 7 . Under a normal operation a rated current $I(t)$ flows through the coil equipped in a magnet relay (RL-33E, YASUKAWA). The differential $\mathrm{d} I(t) / \mathrm{d} t$ of $I(t)$ is sensed by a transformer coupling. Then, such a voltage signal $E_{\mathrm{m}}(t)$ that is proportional to $d I(t) / \mathrm{d} t$ is fed to a buffer-filter circuit. Since the PD signal has high frequency components, a High-Pass (HP) filter is placed between a buffer and the LA. A ferrite core used for the Current Transformer (CT) has a frequency limit at higher side. Therefore, with our method, the front end works as a band-pass filter.

Let us see how $E_{\mathrm{m}}(t)$ is proportional to $\mathrm{d} I(t) / \mathrm{d} t$. An enlarged picture of the ferrite core is shown in Fig. 7. Let the radius of the core be $r$ and the thickness of it be $t_{\mathrm{c}}$ where $r \gg t_{\mathrm{c}}$. Magnetic flux $\Phi(t)$ is induced by $I(t)$ along the circumference of the core. This is expressed, when $r \gg t_{\mathrm{c}}$, as

$$
\Phi(t)=\int_{\mathrm{s}} \boldsymbol{B} \cdot \boldsymbol{t} \mathrm{d} S=B S
$$

where $B$ is the flux density induced by $I(t)$ and $S$, the shaded area in Fig. 7, is perpendicular to $\boldsymbol{B}$. In eq. (4. 1) the vector $\boldsymbol{t}$ is a unit vector tangent to the circumference of the core. Let $\mathrm{d} s$ be a tiny vector along $\boldsymbol{B}$. Then Ampere's law gives

$$
\int \boldsymbol{B} \cdot \mathrm{d} \boldsymbol{s}=\mu n_{1} I(t)
$$




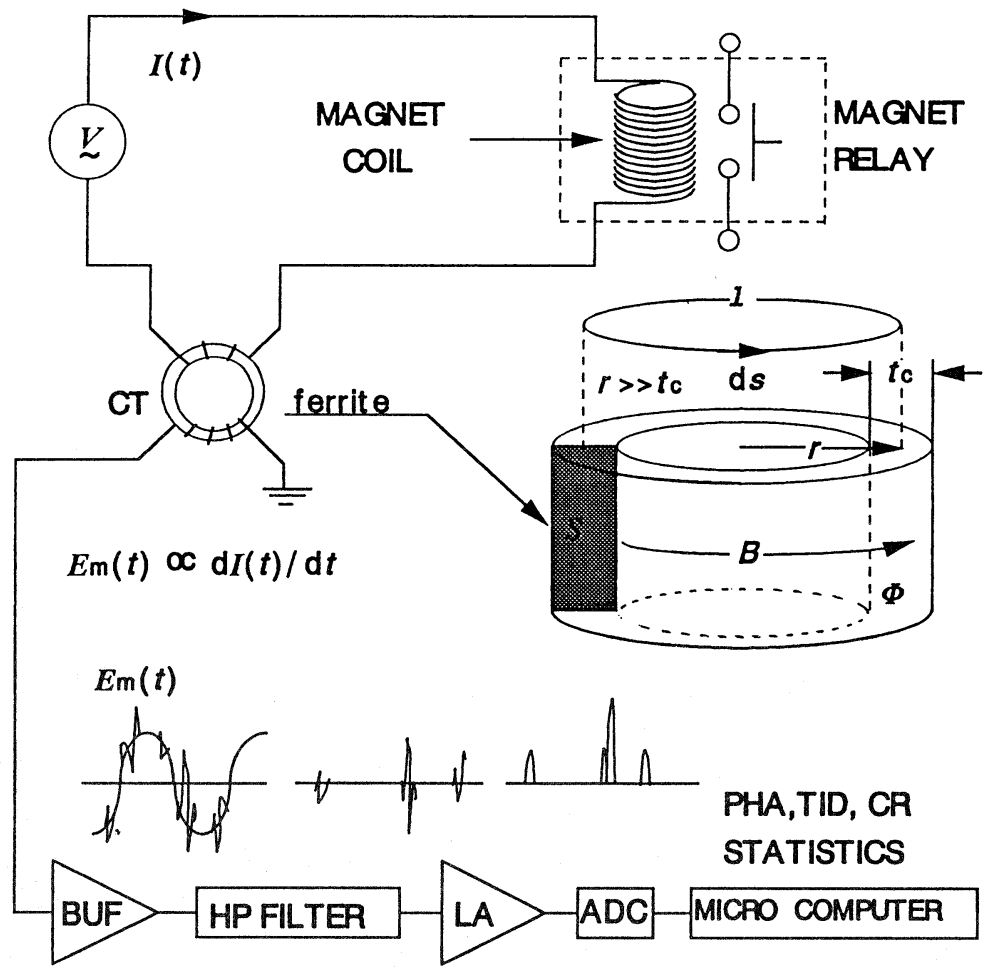

Fig. 7 Schematic illustration of the method in order to pick up PD pulses from a magnet coil.

Where $\mu$ expresses the permeability of the core and $n_{1}$ the winding number in the relay side. Taking the relation $r>t_{\mathrm{c}}$, into account, the left side of the eq.(4.2) becomes

$$
\int B \cdot \mathrm{d} s=B l
$$

where $l$ is the mean length of the circumference. From eqs. (4. 2) and (4. 3)

$$
B l=\mu n_{1} I(t)
$$

Substituting $B$ in eq.(4. 4) for $B$ in eq.(4. 1), $\Phi(t)$ is expressed as

$$
\Phi(t)=\left(\mu S n_{1} / l\right) I(t)
$$

In the secondary side of the CT, an induced voltage $E_{\mathrm{m}}(t)$ appears across the secondary winding. This is written by using Faraday-Lenz's law as

$$
E_{\mathrm{m}}(t)=n_{2}\{-\mathrm{d} \Phi(t) / \mathrm{d} t\}=-\left(n_{1} n_{2} \mu S / l\right) \mathrm{d} I(t) / \mathrm{d} t
$$


where $n_{2}$ is the winding number of CT at secondary side. When $n_{1}$ and $n_{2}$ are kept constant, then $E_{\mathrm{m}}(t)$ above is expressed as

$$
E_{\mathrm{m}}(t)=K \mathrm{~d} I(t) / \mathrm{d} t
$$

by defining a constant $K=-\left(n_{1} n_{2} \mu S / l\right)$. Equation (4.6) means that $E_{\mathrm{m}}(t)$ is proportional to the differential of $I(t)$. By appropriately adjusting the constant $K$ we may pick up tiny PD pulses ${ }^{33}$.

\section{2 Preliminary experiment}

A preliminary and coarse experiment was practiced by using a storage oscilloscope. The result of the experiment is represented in Fig. 8. In this experiment the breakdown process of the coil insulator is accelerated by forcing an overcurrent to flow across the coil. With this procedure the temperature of the coil goes up higher than rated operation because an excess loss due to the overcurrent contributes to the excess generation of heat. The procedure is realized by removing the core of the magnet coil.

A correlation measurement was performed here among the variation of coil temperature, coil current and PD pulse number. The time $t_{e \%}$ elapsed after energizing the coil ${ }^{33)}$ is expressed in normalized one. It is normalized to a breakdown time. Therefore, a full percentage (100\%) in $t_{e \%}$ means that the coil comes to breakdown at $t_{e \%}=100 \%$. The number of PD pulses are manually recorded by using the storage oscilloscope.

In Fig. 8 a tendency is clearly seen that PD pulses appear well before the coil breakdown. PD pulses also continue to appear within the intermediate period of the breakdown process, though the temperature and the current show no appreciable increase during the period. Excessive numbers of PD pulses are observed near the end of the breakdown process. In the same period, both the coil

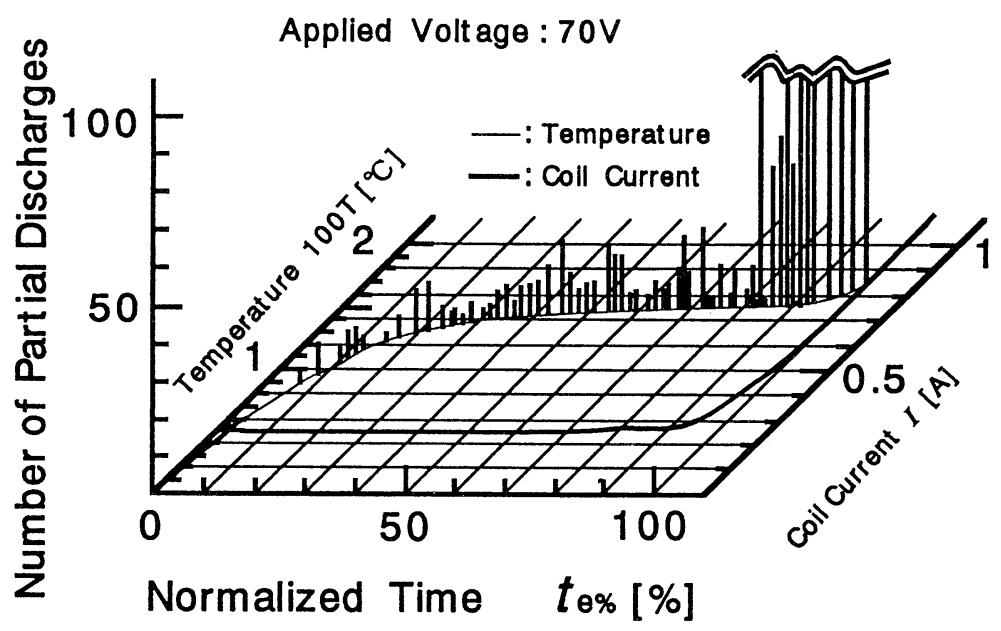

Fig. 8 Observation of the coil breakdown process by coincident measurement of coil temperature, coil current, and PD pulses along $t_{e \%}$, where $t_{e \%}$ expresses the time elapsed after the test voltage application to the coil. The time $t_{e \%}$ is normalized to the breakdown time and $t_{e \%}$ at the breakdown time becomes $100 \%$. 


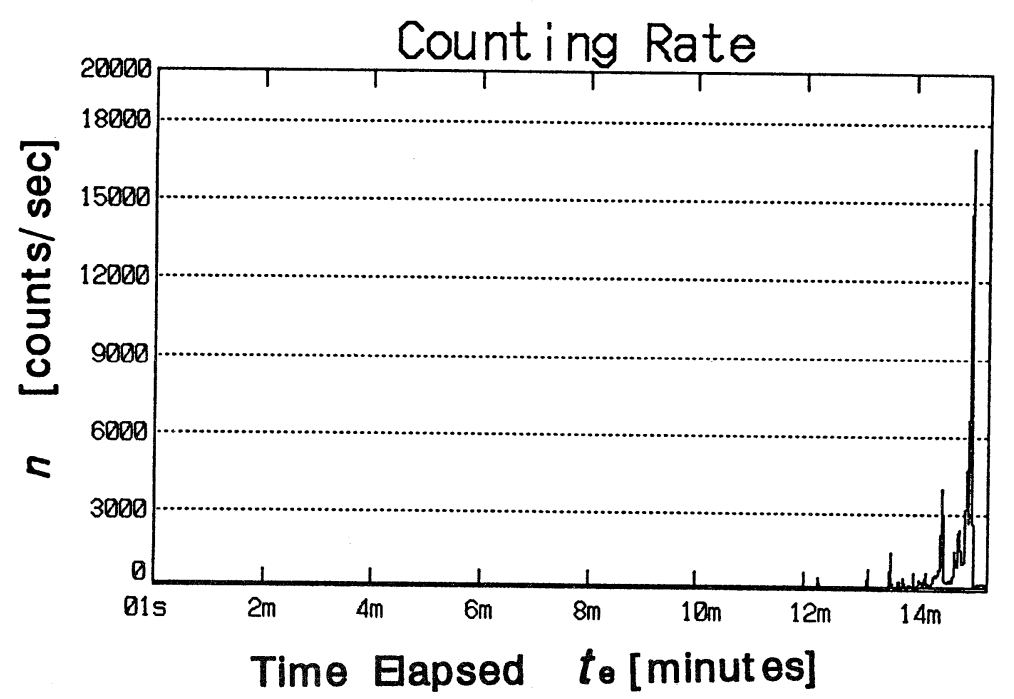

Fig. 9 Observation of the variation of PD pulse numbers with respect to the time elapsed.

temperature and the coil current begin to increase steeply, which is in accordance with the conventional observation $^{29)}$. The fact shown in Fig. 8 gives us a basis of employing a PD pulse train as a means to anticipate the coil breakdown.

\section{3 Observation by the computer system}

\section{3. 1 Counting rate variation of $P D$ pulses}

With our system we can derive a CR expression. The unit time for CR data arrangement can be chosen arbitrarily. An example result is shown in Fig. 9. In Fig. 9 it is evident that the CR of PD pulses increases remarkably near the end of the brekdown process. This resit coincides with the one represented in Fig. 8.

\section{3. 2 Variation of pulse height distribution}

As was mentioned in $\S 4$. 1, the pulse height of a PD pulse obtained by our system does not correspond directly to the magnitude of a PD. However, as expressed in eq.(4. 6), a pulse height in our experiment is regarded as a measure of PD magnitude ${ }^{20)}$. Therefore, the variation of PHD indicates the information concerning the deterioration of coil insulation. The PHD variation against the elapsed time $t_{\mathrm{e}}$ is represented in Fig. 10. It should be noted here that, as was mentioned in $\S 3, t_{\mathrm{e}}$ is expressed not by time itself but by cumulative count $N_{\mathrm{p}}$ of PD pulses. The deterioration of insulator in a magnet coil is thought to depend on the history of PD pulse generation. Therefore in Fig. 10, the temporal variation of PHD is displayed along $N_{\mathrm{p}}$ axis.

\section{3. 3 Observation of deterioration process by TID}

As has been stated in $\S 1$ we can check the independence of each pulse by TID observation ${ }^{22,24)}$. In our system a TID representation is reconstructed from the original pulse train. A result is shown in Fig. 11. It it is seen that pulses appearing in the course of deterioration do not show complete independence to each other because the TID curve no more represents an exponential variation ${ }^{24)}$. 




Fig. 10 Variation of PHD with respect to the elapsed time. It should be noted that $t_{e}$ is expressed not by the time itself but cumulative count $N_{\mathrm{p}}$.



Fig. 11 Investigation by TID on the independence of PD pulses which appear in the course of deterioration. 


\section{3. 4 Outlook of individual pulses}

The rate of pulse occurrence varies rapidly up and down in an occasion. In that case a PHD or a TID loses its sense. That is because we assume a stationary process when we derive a PHD or a TID. If the occurrence of the pulse is not from a stationary process, the PHD or the TID has no more its sense. In case an occurrence rate changes remarkably, the phenomenon is not said to be a stationary process. Instead it should be regarded as a mixed phenomenon consisting of different properties. In such an occasion another method for observing the property should be proposed. One way we attempted is to trace and display the individual pulses of a pulse train. An example of the result is illustraed in Fig. 12. In this description we can see at the same time, concerning a pulse, the pulse height and the occurrence time of each pulse against $N_{\mathrm{p}}$.

In Fig. 12 the occurrence time is represented in two kinds of ways. The one is an immediate representation by the elapsed time $t_{e}$. The other is a representation by cumulative count $N_{\mathrm{p}}$. The

\section{A : 3D-Representation}

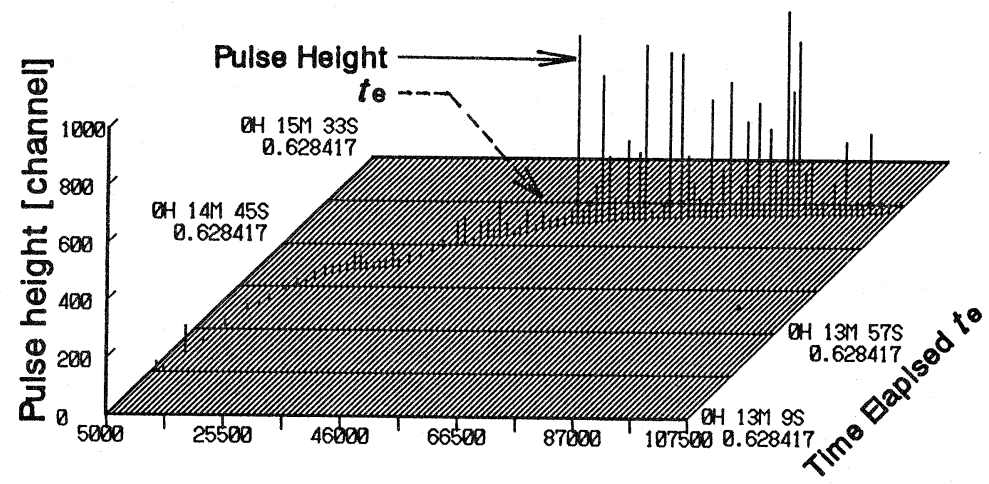

\section{B : 2D-Representation}

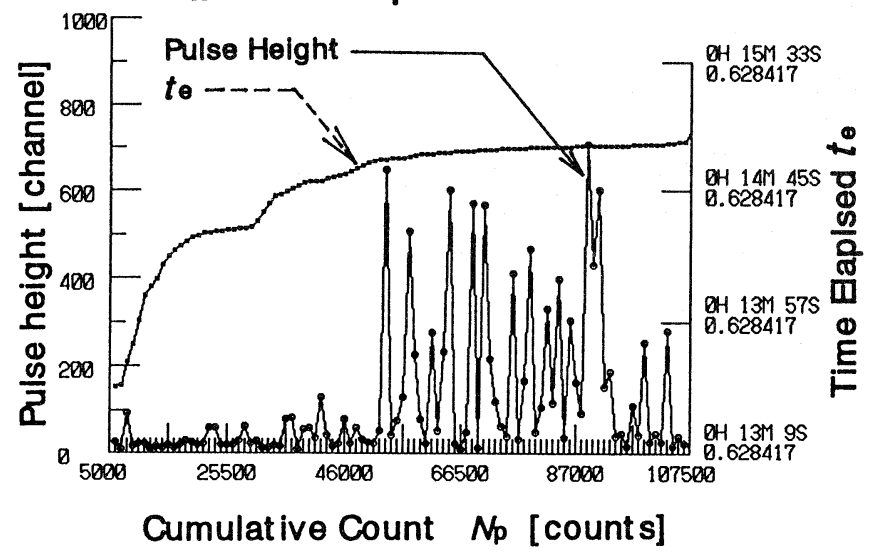

Fig. 12 The outlook of a pulse train by 3D-and 2D-Representation which show the profile of respective pulses in terms of pulse height, occurrence time $t_{\mathrm{e}}$ and cumulative count $N_{\mathrm{p}}$. 
reason we choose both $t_{e}$ and $N_{\mathrm{p}}$ as measures of elapsed time is that the relation between $t_{e}$ and $N_{\mathrm{p}}$ simply shows whether or not the phenomenon is stationary. If a pulse train is an outcome of a stationary process, then, on an average, the count rate is constant. This means that $t_{\mathrm{e}}-N_{\mathrm{p}}$ curve lies along a line, though $t_{e}$ or $N_{\mathrm{p}}$ of each pulse fluctuates around periodic points. The fact that $t_{e}$ $N_{\mathrm{p}}$ curve becomes a line is easily understood if we assume the mean rate $f_{0}$ of the pulse train is constant. This is because the mean interval $\tau=1 / f_{0}$ of the pulse train combines $t_{e}$ and $N_{\mathrm{p}}$ as such that $t_{e}=N_{\mathrm{p}} \times \tau$. However, the $t_{e}-N_{\mathrm{p}}$ curve shows several tangent lines when the pulse train consists of various parts having different count rates. The example of that can be seen in Fig. 12-A, a 3D-Representation. By 3D-Representation the pulse height of each pulse is displayed as well on the $t_{e}-N_{\mathrm{p}}$ curve. The variation of $t_{e}-N_{\mathrm{p}}$ curve in detail can be seen in 2D-Representation as illustrated in Fig. 12-B.

\section{3. 5 Statistical treatment}

It has already been shown that statistical analyses were useful on the insulation diagnosis ${ }^{10,11,15)}$. Therefore, we also added to our system such functions as to derive frequency distributions $f(n)$ of pulse number $n$, where $n$ is a pulse count per unit time $t$ which is represented in Fig. 6. Once $f(n)$ is constructed from a pulse train, then we can extract such statistical moments ${ }^{34)}$ as the mean $\mu$, the variance $\sigma^{2}$, the skewness $S$ and the kurtosis $K$ of the distribution $f(n)$. An example of the variation of each statistical quantity against $t_{e \%}$ is represented in Fig. 13. In Fig. 13, $t_{e \%}$ is the elapsed time expressed in \% and is normalized to the breakdown time of the coil insulator as was stated in $\S 4$. 2. Therefore, $100 \%$ $t_{e \%}$ is the time at which the insulator breakdown takes place. In Fig. 13, $\mu, \sigma, S$ and $K$ are calculated every $10 \% t_{e \%}$. It is seen in the figure that, near the end of the breakdown process, $\mu$, $\sigma, S$ and $K$ deviate from the ones residing in the intermediate range of the breakdown process. With the present sample $\mu, \sigma$, and $K$ show appreciable deviations. In case a quantity represents a variation which coincides with $t_{e \%}$, we can choose that quantity as a measure of the anticipation of an insulator. In the present example we may anticipate the breakdown by $\mu$, $\sigma$, and $K$.

As was described in $\S 3$, we also attached a statistical test function to our system. With this test we can check the difference of two adjacent $f(n)$ s along $t_{e \%}$. The mean difference test and the test on variance are included in the

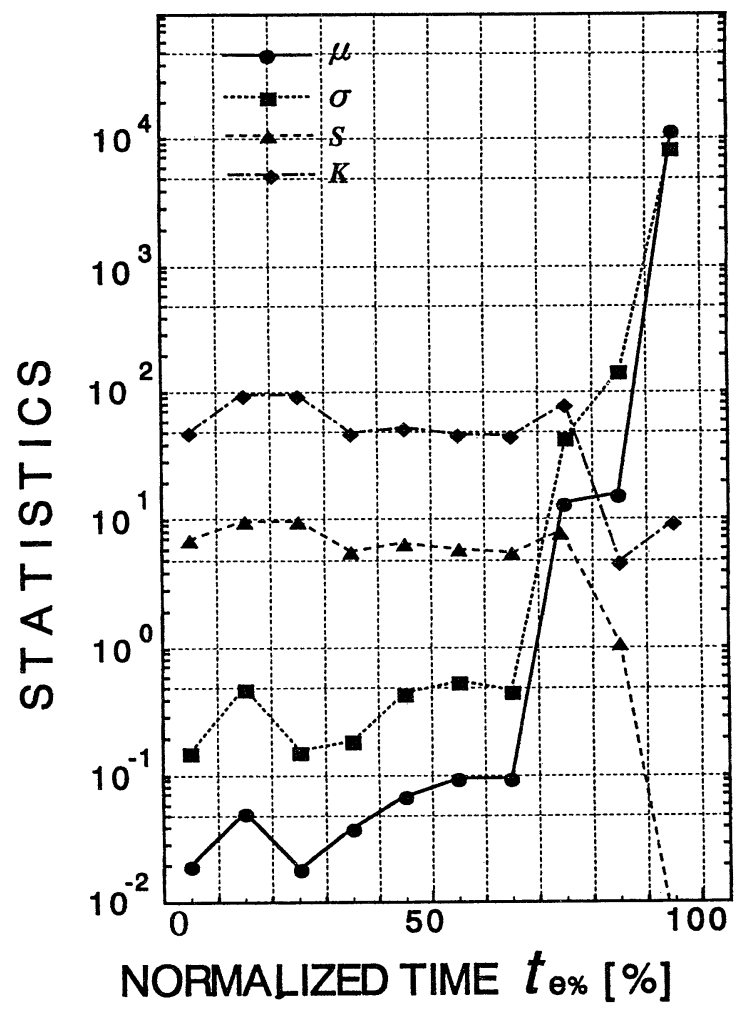

Fig. 13 The variation of statistics obtained from pulse number distribution $f(n)$ s along the elapsed time $\boldsymbol{t}_{\mathrm{e} \% \text {. }}$ 
system. When we can not recognize an appreciable difference between two adjacent $f(n)$ s, the investigation of the discrepancy by a statistical test is useful because the test gives us a judgement basis whether of not the breakdown process is progressing.

\section{Conclusion}

A pulse train measurement and analysis system based on a commercially available Wilkinson ADC and a hand-made interface (IF) board in connection with a micro computer (PC9801VM21, NEC) was described. The dead time of our system is the interval between a pulse input time and the time at which the system becomes possible to receive the succeeding pulse. It consists of the time which CPU needs to fetch the ADC data, the handshake time between the IF and the ADC, and the conversion time of the ADC. In the present setup the handshake time is less than $15 \mu s$ and the ADC conversion time is $0.02 \times$ (channel number which corresponds to the pulse height) $\mu s$. This means that the system dead time does not exceed $35.5 \mu \mathrm{s}$ because the maximum channel number of the ADC is 1024 and this gives the longest dead time $20 \mu s=1024 \times 0.02 \mu s$. The setup we proposed here is a software-burdened system, and we can extract such useful pieces of information as PHD, CR, TID, 3D-and 2D-Representation of respective pulses, pulse number distribution $f(n)$, and various moments of $f(n)$. All of the derived quantities are able to be represented with respect to the elapsed time. The 3D-and 2D-Representation are especially helpful when the mean rate of the pulse occurrence varies rapidly. The remarkable feature of the proposed system is that it can observe a long history of a pulse train in order to investigate the aging of the pulse source. With the present construction, the system can trace analog pulses up to about $10^{10}$ counts. A bank RAM and a hard disk memory was cleverly used in order to realize the performance.

The system was applied to the anticipation of a magnet coil breakdown. From the analysis using CR, PHD, TID, 3D-and 2D-Representation, and $f(n)$, the system proved to be applicable to the anticipation. The characteristic of the temporal variation of the pulse occurrence rate coincided well with the one viewed by the load current or the ambient temperature of the coil.

\section{Acknowledgement}

The authors appreciate the dedication and the support of Mrs. M. Sugawara and Mr. Y. Takahashi to this work.

\section{References}

1) see, for example, G.F.Knoll : Radiation Detection and Measurement (John Wiley \& Sons, New York, 1979) Chap.3, pp.95-102.

2) John P. Yangos and S.-H. Chen : "Simple Low-Cost Digital Events Analyzer" Rev. Sci. Instrum. vol.51, pp.334-350 (1980).

3) W. Niiyama and K. Miyakawa : "Simple Low-Cost Time-Interval Analyzer" Jpn. J. Appl. Phys. vol.24, pp.1561-1564 (1985).

4) T. Akimoto, I. Murai, A. Chaki, Y. Ogawa and I. Shoji : "A Dual-Parameter Multichannel Analyzer Using a Personal Computer” IEEE Trans. Nucl. Sci. vol.36, pp.1354-1358 (1985).

5) C. Imperiale : "A Fast Versatile Single Dual-Parameter Multichannel Analyzer" IEEE Trans. Nucl. Sci. vol.39, pp.283-289 (1992). 
6) T. Akimoto, I. Murai, S. Kato, S.Chaki, I. Shoji and Y. Ogawa : "A Dual-Parameter Multichannel Analyzer by a Dual-Computer System” IEEE Trans. Nucl. Sci. vol.37, pp.15941598 (1990).

7) S. Kobayashi and K. Goshima: "Apparatus for Measuring Time Intervals of the Beating Rhythm of Cultured Cardiac Cells" Rev. Sci. Instrum. vol.55, pp.1691-1695 (1984).

8) H. Sonoda, K. Fujii, H. Sato, T. Oshio and N. Suzuki : "A 1024-channel Digital Apparatus for Measurements of the Time Interval Distribution” Jpn. J. Appl. Phys. vol.7, pp.1405-1408 (1978).

9) R. Bartnikas : "Use of a Multichannel Analyzer for Corona Pulse-Height Distribution Measurements on Cables and Other Electrical Apparatus" IEEE Trans. Instrument. and Measurement vol.IM-22, pp.403-407 (1973).

10) J. Austin and R. E. James : "On-Line Digital Computer System for Measurement of Partial Discharges in Insulation Structures” IEEE Trans. Electr. Insulation vol.El-11, pp.129-138 (1976).

11) T. Tanaka and T. Tatsuki : "A Minicomputer-Based Partial Discharge Measurement System" Conf. Rec. 1978 IEEE Int. Symp. on Electr. Insulation, 1978 pp.86-89 (1978).

12) J. Macur and K. Domansky: "Distribution of Amplitudes and Time Intervals between Partial Discharges" Jpn. J. Appl. Phys. vol.30, pp.860-861 (1991).

13) M. G. Phillips, F. L. Guild and B. Hawis: "Acoustic Emission Monitoring of GRP and GRP Structures” Plast. Rubber. Processes Appl. vol.4, pp.1-5 (1984).

14) E. Gulski and F. H. Kreuger : "Digital Computer System for Measurement of Partial Discharges in Insulation Structures" Proc. 3rd Int. Conf. on Conduction and Breakdown in Solid Dielectrics, Trondheim, 1989, p582 (1989).

15) E. Gulski, P.H.F.Morshuis and F.H.Kreuger : "Automized Recognition of Partial Discharges in Cavities” Jpn. J. Appl. Phys. vol.29, pp.1329-1335 (1990).

16) T. Uemura, M. Fujii, R. Igarashi and Y. Narita : The 26th Annual Meeting on Radioisotopes in the Physical Sciences and Industries, Tokyo, July, 1989, 3p-I-3.

17) R. Igarashi, T. Uemura and Y. Narita : Ext. Abstr. 51st Autumn Meeting of the Japan Society of Applyed Physics, Morioka, September, 1990, 28p-MB-2.

18) S.A.Boggs : "Partial Discharge-Part2 : Detection Sensitivity" IEEE Electrical Insulation Magazine vol.6, pp.35-43 (1990).

19) S.A.Boggs and G.C.Stone : "Fundamental Limitations in Measurement of Corona and Partial Discharge" IEEE Trans. Electr. Insulation vol.El-17, pp.143-150 (1982).

20) F.H.Kreuger : Partial Discharge Detection in High Voltage Equipment (Butterworths, London, 1989).

21) G.C.stone : "Partial Discharge-Part7 : Techniques for Measuring PD in Operating Equipment" IEEE Electrical Insulation Magazine vol.1, pp.9-19 (1991).

22) K. Watanabe : "D. C. Partial Dischage and Current in Polyethylene” Jpn. J. Appl. Phys. vol.17, pp.483-490 (1978).

23) J. Macur, K. Domansly and J.Sikula : Stochastic Character of Partial Discharges in Insulators” J. Appl. Phys. vol.67, pp.540-543 (1990).

24) J.L.Putman : "Analysis of Spurious Counts in Geiger Counters" Proc. Phys. Soc. vol.61, pp.312- 
319 (1948).

25) E. P. de Lima, M. Salete, S. C. P. Leite, A. J. P. L. Policapo and M. A. F. Alves : "Dead Region Time Evolution and Afterpulses Development in SQS” IEEE Trans. Nucl. Sci. vol.NS-31, No.1, pp.94-98 (1984).

26) B. A. Bushaw, T. J. Whitaker, B. D. Cannon and R. A Warner: "Time Interval Distribution and Photon-Burst-Correlation Spectroscopy of a Strontium Atomic Beam" J. Opt. Soc. America vol.B2, pp.1547-1553 (1985).

27) S. Takeuchi, T. Nagai, K. Hasegawa and Y. Hosono: "High Performance Random Pulser Based on Photon Counting" IEEE Trans. Nucl. Sci. vol.33, No.1, pp.946-949 (1986).

28) S. Guldbakke and H. Klein : "Dead Time of Geiger-Muller Photon Dosimeters" Rep. Comm. Eur. Commun. vol.EUR-7448-2, pp.385-394 (1981).

29) G. C. Stone and S. A. Boggs: "Propagation of Partial Discharge Pulse in Shielded Power Cable" 1982 Ann. Rep. Conf. on Electrical Insulation and Dielectric Phenomena, U.S. National Academy of Science, 1982, pp.275-280 (1982).

30) B.F.Hampton and R.J.Meats : "Diagnostic Measurement at UHF in Gas Insulated Substations" IEE Proc., Part C vol.135, pp.137-144 (1988).

31) P.H.F.Morshuis and F.H.Kreuger: "Discharge Phenomena in Voids Filled With Fibrous Materials" Proc. 3rd Int. Conf. on Conduction and Breakdown in Solid Dielectrics, Trondheim, 1987, pp.117-121 (1987).

32) H.J.Weber, R.E.Sceberger and G. Stolpe : Field Measurements of Partial Discharges in Potential Transformers" IEEE Electr. Insul. Magazine vol.2, pp.34-38 (1986).

33) T. Ishida, Y. Mizuno, M. Nagao and M. Kosaki : "Swarming Pulsive Microdischarge Characteristics in Internal Void of Epoxy Resin" Int. conf. Materials Engng. for Resources. ICMR '91 AKITA, 1991 pp.19-20 (1991).

34) see, for example, M.R.Spiegel : Probability and Statistics, (McGraw-Hill, London, 1980) chap. 4. 